\title{
EFEITO DE DOSES DE NITROGÊNIO SOBRE NEMATOIDES DO ALGODOEIRO EM CAMPO
}

\author{
ROSSI, Carlos Eduardo ${ }^{1}$ \\ CARVALHO, Luiz Henrique ${ }^{1}$ \\ NEVES, Samira Scaff ${ }^{2}$ \\ AGUIAR, Adriano Tosoni da Eira ${ }^{1}$
}

\begin{abstract}
RESUMO: Um experimento foi instalado em campo naturalmente infestado por Rotylenchulus reniformis e Pratylenchus brachyurus com o objetivo de verificar o efeito do nitrogênio (N) sobre esses nematoides em algodoeiro. O delineamento estatístico foi blocos ao acaso com parcelas subdivididas com 4 níveis de $\mathrm{N}$ nas parcelas (ureia: 16, 64, 144 e $256 \mathrm{~kg} \mathrm{~N}^{-1}$ ) e 3 cultivares de algodão (IPR 02-307, Delta Opal e NuOpal) nas subparcelas. Os nematoides foram extraídos de amostras de solo e raízes, identificados e estimadas suas densidades populacionais. Nenhuma dose de $\mathrm{N}$ afetou as densidades populacionais dos dois nematoides e não se observaram diferenças estatísticas entre as cultivares estudadas, apesar de IPR 02-307 e NuOpal demonstrarem 47,4 e 38,4\%, respectivamente, menos nematoides da espécie Pratylenchus brachyurus do que Delta Opal.
\end{abstract}

Palavras-chave: Gossypium hirsutum. Pratylenchus brachyurus. Rotylenchulus reniformis. Sistema adensado. Fertilizante.

\section{EFFECT OF NITROGEN RATES ON THE COTTON NEMATODES IN FIELD}

SUMMARY: A field experiment was conducted in naturally infested with Pratylenchus brachyurus and Rotylenchulus reniformis in order to verify the effect of nitrogen $(\mathrm{N})$ on these nematodes in cotton. The statistical design was randomized blocks with split plot design with four levels of $\mathrm{N}$ in the plots (urea: $16,64,144$ and $256 \mathrm{~kg}$ $\mathrm{N} \mathrm{ha}^{-1}$ ) and three cotton cultivars (IPR 02-307, Delta Opal and NuOpal) subplots. The nematodes were extracted from samples of soil and roots, identified and their densities estimated. No amount of $\mathrm{N}$ affected the population densities of both nematodes and there were no statistical differences between cultivars, although NuOpal and IPR 02307 show 47.4 and $38.4 \%$, respectively, less Pratylenchus brachyurus than Delta Opal.

Keywords: Gossypium hirsutum. Pratylenchus brachyurus. Rotylenchulus reniformis. Narrow spacing. Fertilizer.

\section{INTRODUÇÃO}

O efeito dos nematoides pode limitar a produção de algodão. Estimam-se prejuízos médios de 10,7\% causados por eles nessa cultura (SASSER; FRECKMAN, 1987). As espécies predominantes são Meloidogyne incognita raças 3 e 4, Rotylenchulus reniformis e, mais recentemente, Pratylenchus brachyurus (ROBINSON, 2008). Como o algodão é cultivado em áreas extensivas, as medidas de controle restringem-se a rotação de culturas com plantas não hospedeiras, a resistência de cultivares e o uso de nematicidas (ASMUS, 2009).

Fatores bióticos e abióticos como presença de outros patógenos ou agentes de controle biológico, temperatura, umidade, estrutura do solo, fertilidade interferem na relação dos nematoides com o algodoeiro, aumentando ou diminuindo o dano (NORTON; NIBLACK, 1991). Os fertilizantes e corretivos modificam a fertilidade do solo e a planta qualitativamente, o que pode influenciar os nematoides, seja pela maior disponibilidade de sítios de alimentação ao parasito, quando há

\footnotetext{
${ }^{1}$ Instituto Agronômico

${ }^{2}$ UNESP/FCAV
} 
correspondente aumento de volume radicular, ou pelas alterações fisiológicas induzidas pelos diferentes nutrientes minerais. Além disso, podem alterar a composição e a densidade microbiana na rizosfera interferindo no comportamento dos parasitos, podendo em alguns casos controlá-los (YEATES, 1987; VESTERGARD, 2004).

Com relação ao nitrogênio (N), Rodríguez-Kábana (1986) revisou as informações na literatura e relata que fertilizantes inorgânicos contendo nitrogênio amoniacal, como a ureia, dependendo da quantidade, podem suprimir nematoides, como exemplos Rotylenchulus reniformis em oliveiras, Mesocriconema xenoplax em ameixeiras e Tylenchulus semipenetrans em citros. Esse fenômeno pode variar conforme a espécie de nematoide e a planta hospedeira. Melakeberhan (2006) observou efeito supressivo de fertilizante nitrogenado nas densidades populacionais de $M$. incognita e P. penetrans em soja suscetível a Heterodera glycines e somente para $M$. incognita em cultivar tolerante. Já na cultivar resistente, a fertilização aumentou $P$. penetrans. Este estudo testava a hipótese da eficiência do uso de fertilizante (EUF), ou seja, a quantidade de fertilizante a ser aplicada cuja resposta proporcionaria aumento na produtividade da planta e/ou decresceria a densidade populacional do nematoide.

Outras características químicas do solo podem relacionar-se com as populações de nematoides. Sologuren; Santos (1997) e Pinheiro et al. (2008) comprovaram a influência da composição química dos solos (cálcio, potássio, magnésio e pH) na densidade populacional e distribuição espacial de $H$. glycine.

$\mathrm{Na}$ cultura do algodoeiro, Silva et al. (1997) demonstraram que o aumento de $\mathrm{N}$ na forma de ureia aplicado nessa planta diminui os sintomas reflexos ("carijó") e aumenta sua capacidade produtiva em locais infestados por nematoides.

$\mathrm{O}$ objetivo desse trabalho foi verificar o efeito do $\mathrm{N}$ em diferentes doses em três cultivares de algodão cultivadas em sistema adensado sobre as populações de nematoides em área naturalmente infestada.

\section{MATERIAL E MÉTODOS}

O ensaio foi realizado no município de Leme (SP). O solo da área foi classificado como Latossolo vermelho distrófico típico (EMBRAPA, 2006), A moderado, textura argilosa, álico (MO: $33 \mathrm{~g} \mathrm{dm}^{-3}, \mathrm{pH}$ : 5,5, P: $34 \mathrm{mg} \mathrm{dm}{ }^{-3}, \mathrm{~K}: 2,2 \mathrm{Mg}: 21, \mathrm{H}+\mathrm{Al}: 28$, SB: 57,2, CTC: $85 \mathrm{mmol}_{\mathrm{c}} \mathrm{dm}^{-3} \mathrm{e} \mathrm{V}: 67 \%$ ), além de apresentar histórico de infestações por nematoides (Machado et al., 2005b). O delineamento estatístico foi fatorial em blocos ao acaso com parcelas subdivididas com tratamentos qualitativos e quantitativos e fator A: 4 níveis de $\mathrm{N}$ nas parcelas (ureia: 16, 64, 144 e $256 \mathrm{~kg} \mathrm{~N}$ ha $^{-1}$ ) e fator B: 3 cultivares de algodão (IPR 02-307, Delta Opal e NuOpal) nas subparcelas e 4 repetições. Realizou-se uma adubação de plantio com $400 \mathrm{~kg} \mathrm{ha}^{-1}$ de 4-20-20 + $20 \mathrm{~kg} \mathrm{ha}^{-1}$ de Ca; $0,5 \mathrm{~kg} \mathrm{ha}^{-1} \mathrm{de} \mathrm{B} ; 6 \mathrm{~kg} \mathrm{ha}^{-1}$ de Mn e $3 \mathrm{~kg} \mathrm{ha}^{-1} \mathrm{de} \mathrm{Zn.} \mathrm{A} \mathrm{Tabela} 1$ mostra as diferentes doses de ureia (tratamentos) e épocas de aplicação em cobertura. O espaçamento foi $0,45 \mathrm{~m}$ com 8 plantas $\mathrm{m}^{-1}$ (sistema adensado). A parcela totalizava $27 \mathrm{~m}^{2}$. Coletaram-se 5 sistemas radiculares e $1 \mathrm{~L}$ de solo da região próxima à essas raízes, nas duas linhas contíguas às centrais em cada subparcela (TIHOHOD, 1993). As amostras foram encaminhadas ao Laboratório, onde foram processados 5 gramas de raízes pelo método de Coolen; D’Herde (1972) e $200 \mathrm{~cm}^{3}$ de solo por Jenkins (1964). As suspensões contendo os nematoides foram submetidas ao aquecimento a $60^{\circ} \mathrm{C}$ por 3 minutos em banhomaria e, em seguida, fixadas em formol (2\%). As espécies dos nematoides foram identificadas, após montagem de exemplares em lâminas temporárias, por meio de confronto de caracteres morfométricos observados com os descritos em chaves dicotômicas (HANDOO; GOLDEN, 1989; ROBINSON et al., 1997). As densidades populacionais foram estimadas em lâminas de contagem de Peters em microscópio biológico. Esses dados foram analisados pelo programa Sisvar (FERREIRA, 2008) aplicando-se o teste F 
na análise de variância e o teste de separação de médias de Tukey a 5\% de probabilidade de erro.

Tabela 1 - Doses e épocas de aplicação de ureia em algodão $\left(\mathrm{Kg} \mathrm{ha}^{-1}\right)$. Leme, SP. 2009.

\begin{tabular}{cccc}
\hline Doses de ureia & Plantio & $\begin{array}{c}\mathbf{1}^{\mathbf{a}} \text { Cobertura } \\
\text { 30 DAE* }\end{array}$ & $\begin{array}{c}\mathbf{2}^{\mathbf{a}} \text { Cobertura } \\
\text { 45 DAE }\end{array}$ \\
\hline 16 & 16 & - & - \\
64 & 16 & 48 & - \\
144 & 16 & 96 & 32 \\
256 & 16 & 144 & 96 \\
\hline
\end{tabular}

*DAE: dias após emergência

\section{RESULTADOS E DISCUSSÃO}

As espécies de nematoides que ocorreram na área foram Pratylenchus brachyurus com densidades populacionais médias de 44, $6 \mathrm{~g}^{-1}$ raízes e Rotylenchulus reniformis $6,2 \mathrm{~cm}^{-3}$ solo. Para P. brachyurus, essa média obtida estava bem abaixo do limiar de dano que é acima de 9.000 exemplares por planta (INOMOTO et al., 2001). No caso de $R$. reniformis, a média estava dentro da faixa de limiar de dano que é de 1 a 10 espécimes de $R$. reniformis $\mathrm{cm}^{-3}$ solo (ROBINSON, 2007). As densidades de $P$. brachyurus em solo e $R$. reniformis em raízes estavam, em mais de $80 \%$ das amostras, abaixo da capacidade detectável estabelecida pelo método de extração, não participando, portanto, da análise dos dados.

Nenhuma dose de $\mathrm{N}$ afetou as densidades populacionais dos dois nematoides (Tabela 2 e Figuras 1 e 2), ou seja, até $256 \mathrm{~kg} \mathrm{~N}^{-1}{ }^{-1}$ não houve controle dos parasitos e também não houve efeito de interação entre os fatores A e B. De acordo com a literatura é conhecido que somente acima de $300 \mathrm{~kg} \mathrm{~N}$ ha ${ }^{-1}$ pode ocorrer supressão das populações de nematoides. Contrapondo essa informação, num trabalho com algodoeiro com doses de 0 a $75 \mathrm{Kg} \mathrm{N}^{-1}$, Silva et al. (1997) observaram que conforme aumentava-se a dose de N, as notas de sintoma reflexo ("carijó"), indicativo do ataque de nematoides, diminuíam e ocorria um aumento da produção de algodão. Entretanto, os autores não correlacionaram esses resultados de notas com as densidades populacionais dos nematoides, o que não evidencia se o adubo nitrogenado foi realmente a causa das baixas notas de sintomas.

A causa do controle dos nematoides pelo $\mathrm{N}$, no caso a ureia, se deve à liberação de amônia na presença de urease no solo, a qual é um composto nematóxico (RODRÍGUEZ-KABANA, 1986). O modo de ação da amônia no controle dos nematoides não está totalmente evidenciado, porém alguns mecanismos estão envolvidos como a ruptura de membranas, eliminação de gradientes de prótons através da membrana e a exaustão da energia química das células (OKA, 2010).

Apesar da importância do $\mathrm{N}$ na obtenção de elevadas produtividades e no condicionamento de melhor qualidade de fibra, não foram incluídas nesse estudo doses acima de $256 \mathrm{~kg} \mathrm{~N} \mathrm{ha}^{-1}$ por serem consideradas excessivas ao recomendado no cultivo do algodoeiro (RAIJ et al., 1996), o que pode causar o decréscimo na produtividade e na qualidade do algodão e ainda estimular o dano causado por pragas (SMITH; COTHREN, 1999; ROSOLEM; MELLIS, 2010).

Tabela 2 - Efeito de doses de N na forma de ureia sobre as populações de Pratylenchus brachyurus e Rotylenchulus reniformis. Leme, SP. 2009.

\begin{tabular}{ccc}
\hline $\begin{array}{c}\text { Doses de } \mathrm{N} \\
\left(\mathrm{kg} \mathrm{ha}^{-1}\right)\end{array}$ & $\begin{array}{c}\text { Pratylenchus brachyurus } \\
(5 \mathrm{~g} \mathrm{de} \text { raízes })\end{array}$ & $\begin{array}{c}\text { Rotylenchulus reniformis } \\
\left(200 \mathrm{~cm}^{3} \mathrm{de} \text { solo }\right)\end{array}$ \\
\hline 16 & $50,7 \mathrm{a}$ & $1242,3 \mathrm{a}$ \\
64 & $86,9 \mathrm{a}$ & $1132,8 \mathrm{a}$ \\
144 & $66,9 \mathrm{a}$ & $1525,5 \mathrm{a}$ \\
256 & $93,1 \mathrm{a}$ & $1061,9 \mathrm{a}$ \\
\hline $\mathrm{CV}(\%)$ & 18,6 & 5,8 \\
\hline
\end{tabular}

Médias seguidas pela mesma letra na coluna não diferem entre si pelo Teste de Tukey a 5\% de probabilidade.

(Dados apresentados são originais, porém para a análise foram transformados em $\log \mathrm{x}$ ) 
Figura 1 - Efeito de doses de nitrogênio (ureia) em algodoeiro sobre as populações de Pratylenchus brachyurus. Leme/SP.

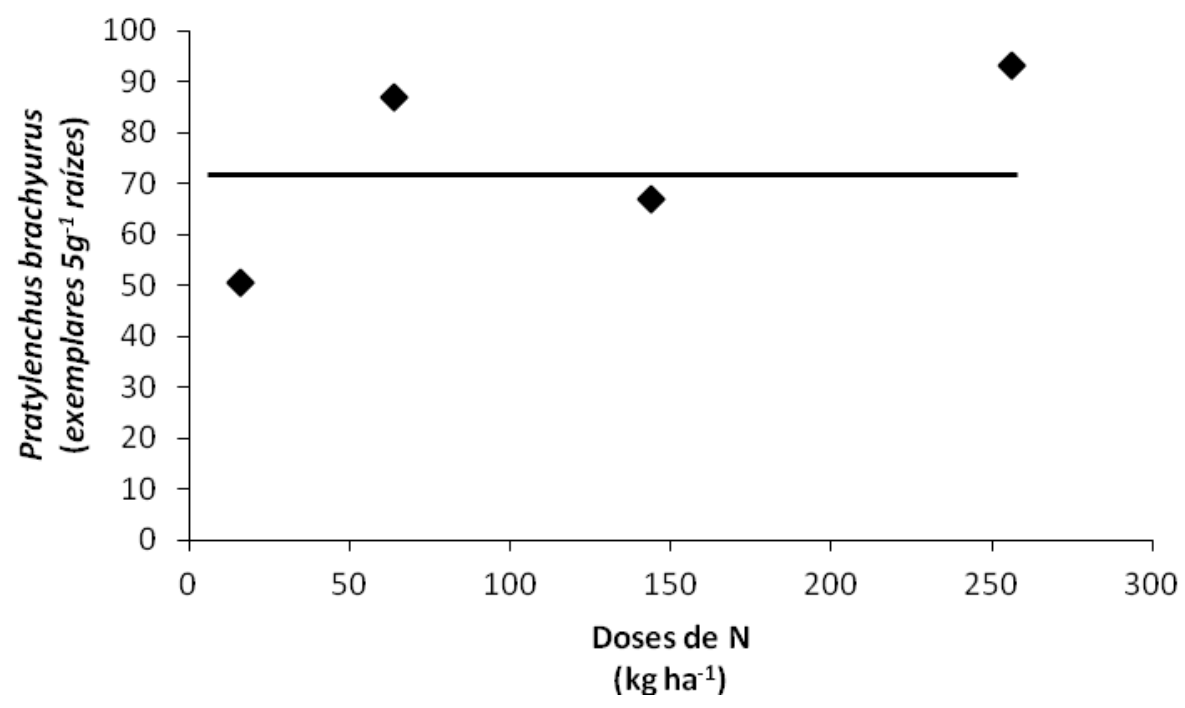

Fonte: Elaborado pelos Autores

Figura 2 - Efeito de doses de nitrogênio (ureia) em algodoeiro sobre as populações de Pratylenchus Brachyurus. Leme/SP.

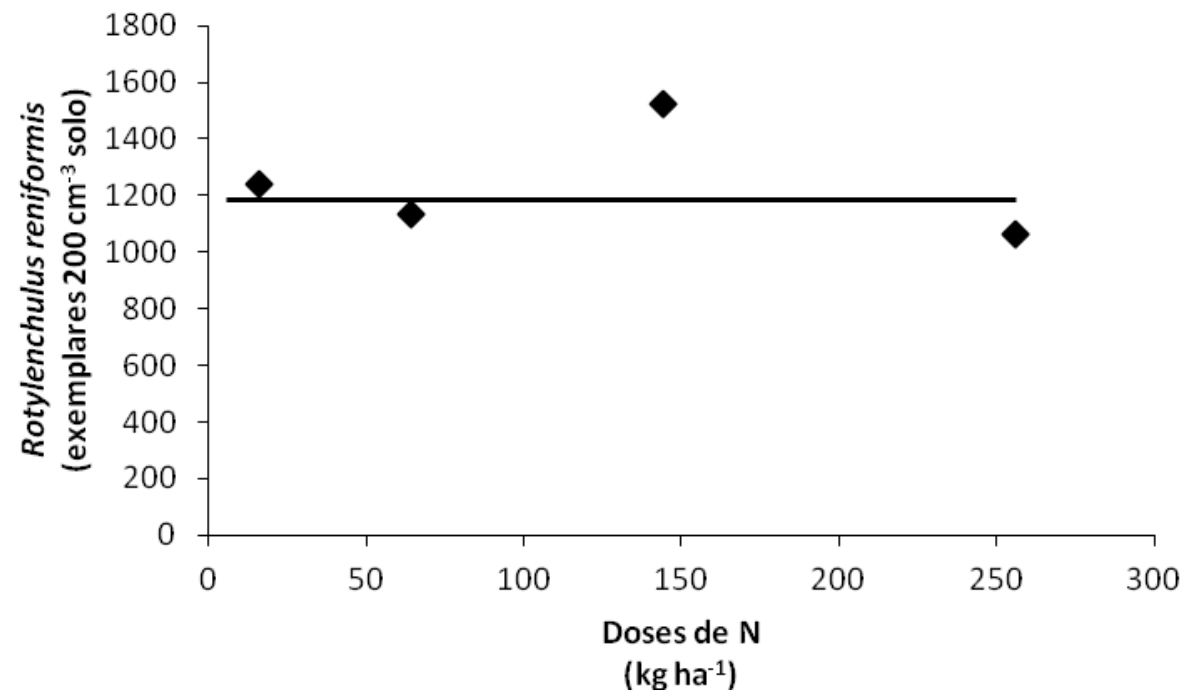

Fonte: Elaborado pelos Autores

Com relação às reações das cultivares estudadas, não se observaram diferenças estatísticas entre elas para os nematoides encontrados (Tabela 3). As três são reconhecidamente suscetíveis a Meloidogyne incognita e Rotylenchulus reniformis (ALMEIDA et al., 2005). Entretanto, IPR 02-307 e NuOpal apresentaram 47,4 e 38,4\%, respectivamente, menos nematoides da espécie Pratylenchus brachyurus do que Delta Opal. Provavelmente, esse fenômeno refere-se a um possível mecanismo de resistência genética dessas duas cultivares em relação a Delta Opal para essa espécie de parasito. Machado et al. (2005a) já haviam verificado a suscetibilidade dessa última cultivar em experimentos com inoculação artificial. 
Tabela 3 - Populações de Pratylenchus brachyurus e Rotylenchulus reniformis em solo e raízes de três cultivares de algodão cultivadas em campo. Leme, SP. 2009.

\begin{tabular}{ccc}
\hline Cultivares & $\begin{array}{c}\text { Pratylenchus brachyurus } \\
(5 \mathrm{~g} \text { de raízes })\end{array}$ & $\begin{array}{c}\text { Rotylenchulus reniformis } \\
\left(200 \mathrm{~cm}^{3} \mathrm{de} \text { solo }\right)\end{array}$ \\
\hline IPR 02-307 & $54,8 \mathrm{a}$ & $1204,8 \mathrm{a}$ \\
Delta Opal & $104,2 \mathrm{a}$ & $1121,1 \mathrm{a}$ \\
NuOpal & $64,2 \mathrm{a}$ & $1396,0 \mathrm{a}$ \\
\hline CV $(\%)$ & 29,0 & 5,1 \\
\hline
\end{tabular}

Médias seguidas pela mesma letra na coluna não diferem entre si pelo Teste de Tukey a 5\% de probabilidade (Dados apresentados são originais, porém para a análise foram transformados em $\log \mathrm{x}$ )

\section{CONCLUSÃO}

A fertilização nitrogenada por meio de ureia em doses variando de 16 a $256 \mathrm{~kg} \mathrm{~N}^{-1} \mathrm{ha}^{-1}$ não tiveram efeito sobre as populações de Rotylenchulus reniformis e Pratylenchus brachyurus nas cultivares IPR 02307, NuOpal e Delta Opal em sistema adensado de cultivo.

\section{REFERÊNCIAS}

ALMEIDA, W. P.; RUANO, O.; MURAMOTO, S.P. Reação de genótipos de algodoeiro (Gossypium hirsutum) a Rotylenchulus reniformis e Meloidogyne incognita raça 3, em casa de vegetação. In: CONGRESSO BRASILEIRO DE ALGODÃO, 5., 2005, Salvador. Anais... Campina Grande: Embrapa Algodão, 2005. (CD-ROM).

ASMUS, G.L. Manejo dos principais nematóides que afetam a cultura do algodoeiro no Brasil. Tropical Plant Pathology, v.34, Suplemento, p.35-36, 2009.

COOLEN, W.A.; D'HERDE, C.J. A method for the quantitative extraction of nematodes from plant tissue. Ghent: State Agriculture Research Center, 1972. 77p.

EMBRAPA - CNPS. Sistema Brasileiro de Classificação de Solos. Brasilia: Embrapa-SPI; Rio de Janeiro: Embrapa-Solos, 2006. 306 p.

FERREIRA, D.F. Sisvar: um programa para análises e ensino de estatística. Revista Symposium, v.6, p.36-41, 2008.

HANDOO, Z.A.; GOLDEN, A.M. A key and diagnostic compendium to the species of the genus Pratylenchus Filipjev, 1936 (lesion nematodes). Journal of Nematology, v.21, p.202-218, 1989.

INOMOTO, M.M.et al. Effect of population densities of Pratylenchus brachyurus on the growth of cotton plants. Fitopatogia Brasileira, v.26, n.2, p.192-196, 2001.

JENKINS, W.R. A rapid centrifugal-flotation technique for separating nematodes from soil. Plant Disease Reporter, v.48,n.6, p.629, 1964.

MACHADO, A.C.Z.; FERRAZ, L.C.C.B.; INOMOTO, M.M. Reação de cultivares de algodoeiro a Pratylenchus brachyurus. In:CONGRESSO BRASILEIRO DE ALGODÃO, 5, 2005, Salvador, BA. Anais... Salvador: Embrapa Algodão, 2005a. CD-ROM.

MACHADO, A.C.Z.; SIQUEIRA, K.M.S.; GALBIERI, R.; CIA, E. Levantamento preliminar das espécies de fitonematóides associadas à cultura do algodão no estado de São Paulo. In: CONGRESSO BRASILEIRO DE ALGODÃO, 5, 2005, Salvador, BA. Anais... Salvador: Embrapa Algodão, 2005b. CDROM. 
MELAKEBERHAN, H. Fertiliser use efficiency of soybean cultivars infected with Meloidogyne incognita and Pratylenchus penetrans. Nematology, v.8, n.1, p.129-137, 2006.

NORTON, D.C.; NIBLACK, T.L. Biology and ecology of nematodes. In: NICKLE, W.R. (ed.). Manual of agricultural nematology. New York: Marcel Dekker, 1991. p.47-72.

OKA, Y. Mechanisms of nematode suppression by organic soil amendments: a review. Applied Soil Ecology, v.44, p.101-115, 2010.

PINHEIRO, J.Bet al. Influência da nutrição mineral na distribuição espacial do nematoide de cisto da soja. Nematologia Brasileira, v.32, n.4, p.270-278, 2008.

RAIJ, B. van et al. Recomendações de adubação e calagem para o Estado de São Paulo. Campinas: IAC, 1996. p.109-111. (Boletim Técnico n 100).

ROBINSON, A.F. Nematode management in cotton. In: CIANCIO, A.; MUKERJI, K.G. Integrated management and biocontrol of vegetable and grain crops nematodes. Dordrecht: Springer, 2008. p.149-182.

ROBINSON, A.F.et al. Rotylenchulus species: identification, distribution, host ranges, and crop plant resistance. Nematropica, v.27, n.2, p.127-180, 1997.

ROBINSON, A.F. Reniform in U.S. Cotton: When, Where, Why, and some remedies. Annual Review of Phytopathology, v.45, p.263-288, 2007.

RODRÍGUEZ-KABANA, R. Organic and inorganic nitrogen amendments to soil as nematode suppressants. Journal of Nematology, v.18, n.2, p.129-135, 1986.

ROSOLEM, C.A.; MELLIS, V. Monitoring nitrogen nutrition in cotton. Revista Brasileira de Ciência do Solo, v.34, p.1601-1607, 2010.

SASSER, J.N.; FRECKMAN, D.W. A world perspective on nematology: the role of the society. In: VEECH, J.A.; DICKSON, D.W. Vistas on nematology. Hyattsville: Society of nematologists, 1987. p. 714.

SILVA, N.M.et al. A adubação nitrogenada e o sintoma de nematóides no algodoeiro. Revista Brasileira de Ciência do Solo, v.21, p.693-697, 1997.

SMITH, C.W.; COTHREN, J.T. Cotton: origin, history, technology, and production. New York: John Wiley \& Sons, 1999. 853p.

SOLOGUREN, L.J.; SANTOS, M.A. Estudo de características químicas de solo em reboleiras de soja com Heterodera glycines. Fitopatologia Brasileira, v.22 (suplemento), p.329, 1997.

TIHOHOD, D. Nematologia Agrícola Aplicada. Jaboticabal: Funep, 1993. 372 p.

VESTERGARD, M. Nematode assemblages in the rhizosphere of spring barley (Hordeum vulgare L.) depended on fertilisation and plant growth phase. Pedobiologia, v.48, p.257-265, 2004.

YEATES, G.W. How plants affect nematodes. Advances in ecological research, v.17, p.61-113, 1987. 